\title{
Bidding process in online auctions and winning strategy: Rate equation approach
}

\author{
I. Yang and B. Kahng \\ School of Physics and Center for Theoretical Physics, Seoul National University, Seoul 151-747, Korea
}

(Received 14 September 2005; published 6 June 2006)

\begin{abstract}
Online auctions have expanded rapidly over the last decade and have become a fascinating new type of business or commercial transaction in this digital era. Here we introduce a master equation for the bidding process that takes place in online auctions. We find that the number of distinct bidders who bid $k$ times up to the $t$ th bidding progresses, called the $k$-frequent bidder, seems to scale as $n_{k}(t) \sim t k^{-2.4}$. The successfully transmitted bidding rate by the $k$-frequent bidder is likely to scale as $q_{k}(t) \sim k^{-1.4}$, independent of $t$ for large $t$. This theoretical prediction is close to empirical data. These results imply that bidding at the last moment is a rational and effective strategy to win in an eBay auction.
\end{abstract}

DOI: 10.1103/PhysRevE.73.067101

PACS number(s): 89.75.Da, 89.65.-s, 89.65.Gh

Electronic commerce (e-commerce) refers to any type of business or commercial transaction that involves information transfer across the Internet. As a formation of e-commerce, the online auction, i.e., the auction via the Internet [1], has expanded rapidly over the last decade and has become a fascinating new type of business or commercial transaction in this digital era. Online auction technology has several benefits compared with traditional auctions. Traditional auctions require the simultaneous participation of all bidders or agents at the same location; these limitations do not exist in online auction systems. Owing to this convenience, "eBay.com," the largest online auction site, boasts over 40 million registered consumers and has experienced rapid revenue growth in recent years.

Interestingly, the activities arising in online auctions generated by individual agents proceed in a self-organized manner [2-7]. For example, the total number of bids placed in a single item or category and the bid frequency submitted by each agent follow power-law distributions [8]. These powerlaw behaviors [9-11] are rooted in the fact that an agent who makes frequent bids up to a certain time is more likely to bid in the next time interval. This pattern is theoretically analogous to the process that is often referred to as preferential attachment, which is responsible for the emergence of scaling in complex networks [12]. This is reminiscent of the mechanism of generating the Zipf law $[10,13,14]$. The accumulated data of a detailed bidding process enable us to quantitatively characterize the dynamic process. In this paper, we describe a master equation for the bidding process. The master-equation approach is useful to capture the dynamics of the online bidding process because it takes into account of the effect of openness and the nonequilibrium nature of the auction. This model is in contrast to the existing equilibrium approach $[15,16]$ in which there is a fixed number of bidders. The equilibrium approach is relevant to traditional auctions; however, it is unrealistic to apply this approach to Internet auctions. The fat-tail behavior of the bidding frequency submitted by individual agents can be reproduced from the master equation. Moreover, we consider the probability of an agent who has bidden $k$ times, called the $k$-frequent bidder, becoming the final winner. We conclude that the winner is likely to be the one who bids at the last moment but who placed infrequent bids in the past.

Our study is based on empirical data collected from two different sources [8]. The first dataset was downloaded from the web, http://www.eBay.com, and is composed of all the auctions that closed in a single day. The data include 264073 auctioned items, grouped into 194 subcategories. The dataset allows us to identify 384058 distinct agents via their unique user IDs. To verify the validity of our findings in different markets and time spans, the second dataset was accumulated over a period of one year from eBay's Korean partner, auction.co.kr. The dataset comprised 215852 agents that bid on 287018 articles in 355 lowest categories.

An auction is a public sale in which property or items of merchandise are sold to the bidder who proposes the highest price. Typically, most online auction companies adopt the approach of English auction, in which an article or item is initially offered at a low price that is progressively raised until a transaction is made. Both "eBay.com" and "auction.co.kr" adopt this rule and many bidders submit multiple bids in the course of the auction. An agent is not allowed to place two or more bids in direct succession. It is important to notice that the eBay auction has a fixed end time: It typically ends a week after the auction begins, at the same time of day to the second. The winner is the latest agent to bid within this period. In such an auction that has a fixed deadline, bidding that takes place very close to the deadline does not give other bidders sufficient time to respond. In this case, a sniper-the last moment bidder-might win the auction, while the bid has a substantial probability of not being transmitted successfully. While such a bidding pattern is well known empirically, no quantitative analysis has been performed on it as yet. In this study we analyze this issue through the rate equation approach.

To characterize the dynamic process, we first introduce several quantities for each item or article as follows:

(i) When a bid is successfully transmitted, time $t$ increases by 1 .

(ii) Terminal time $T$ is the time at which an auction ends. Thus the index of bids runs from $i=1$ to $T$.

(iii) $N(t)$ is the number of distinct bidders who successfully bid at least once up to time $t$. Thus the index of bidders (or agent) runs from $i=1$ to $N(t)$.

(iv) $k_{i}(t)$ is the number of successful bids transmitted by an agent $i$ up to time $t$.

(v) $n_{k}(t)$ is the number of bidders who bid $k$ times 

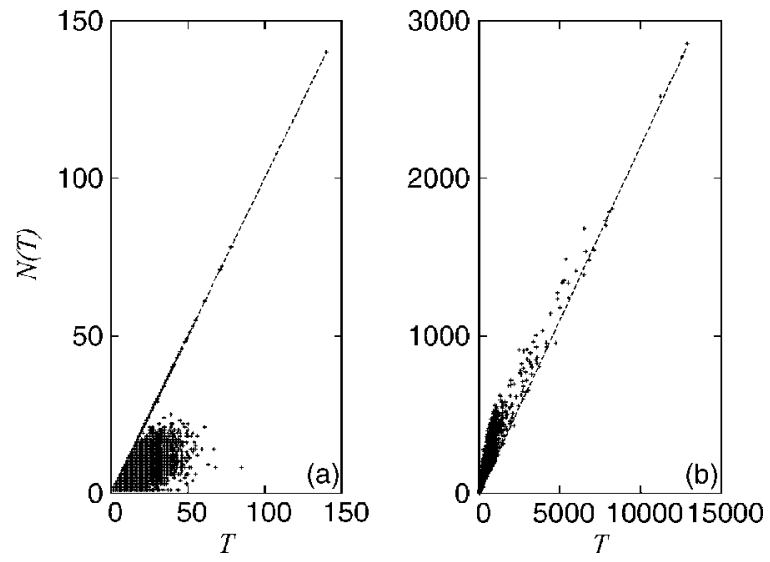

FIG. 1. Plot of $N(T)$ vs $T$ for the eBay (a) and the Korean auction (b), where $N(T)$ is the number of distinct bidders who successfully bid at least once up to terminal time $T$. The dotted lines have slopes of 1 for (a) and 0.22 for (b).

successfully up to time $t$.

From the above, we obtain the relations

$$
N(t)=\sum_{k} n_{k}(t)
$$

and

$$
t=\sum_{k} k n_{k}(t)
$$

for any time $t$ including the terminal time $T$.

It is numerically found that $N(T)$ is likely to increase linearly with increasing $T$, in particular when $T$ is large, for both the eBay and the Korean auction data. When the bidding sequence $T$ is small, $N(T)$ is rather scattered for the eBay data (Fig. 1). In this paper, our interest is focused on the case of large $T$. The proportional coefficient $a$, defined in the relation $N(T)=a T$, is estimated to be $a \approx 1$ for the eBay and $a \approx 0.22$ for the Korean auction data. On the other hand,
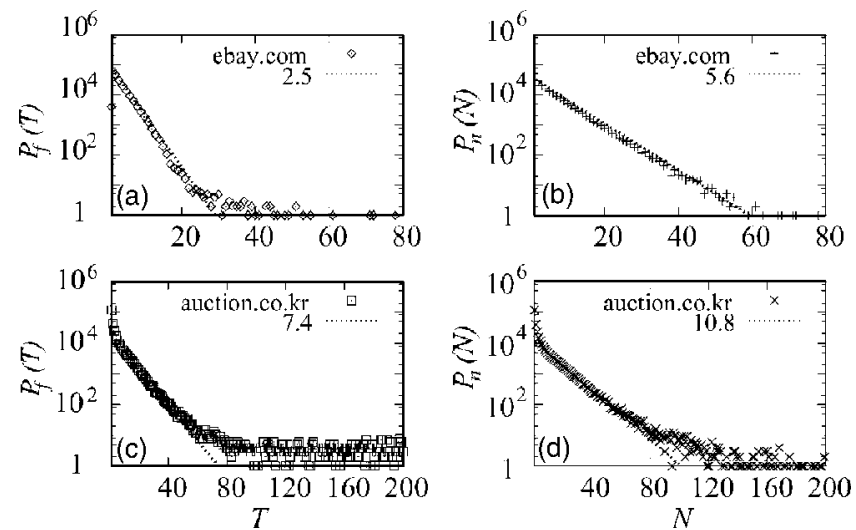

FIG. 2. Plot of $P_{f}(T)$ vs $T$ in (a) and (c), and $P_{n}(N)$ vs $N$ in (b) and $(\mathrm{d})$, where $P_{f}(T)$ is the distribution of total number of bids, and $P_{n}(N)$ is the distribution of total number of participating bidders on each items, for the eBay (a) and (b) and the Korean auction (c) and (d) in semilogarithmic scale. The dotted lines have slopes of 2.5 in (a), 5.6 in (b), 7.4 in (c), and 10.8 in (d).
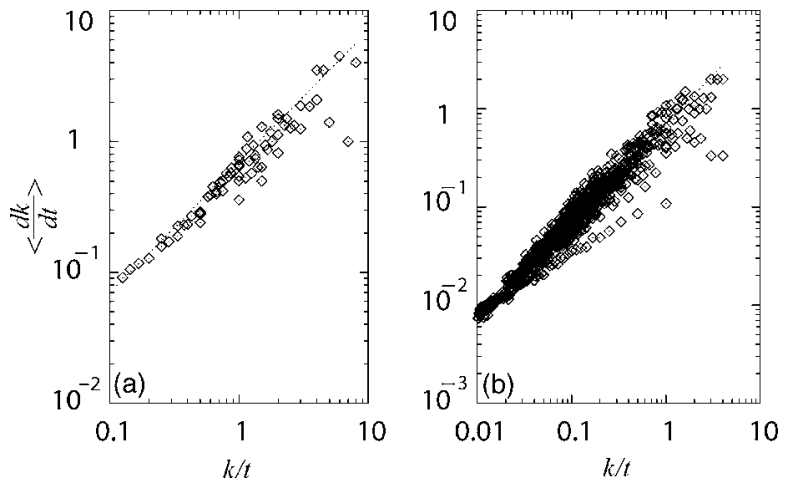

FIG. 3. Plot of the transition rate $\langle d k / d t\rangle$ averaged over different items vs $k / t$ for the eBay (a) and for the Korean auction (b). The dotted lines, obtained by the least square fit in the range [0.1:1] for (a) and $[0.01: 1]$ for (b), respectively, fit to the formula, $\approx 0.7 k / t$ for both data.

the bidding frequencies and the number of bidders for each article are not uniform. Their distributions, denoted as $P_{f}(T)$ and $P_{n}(N)$, respectively, follow the exponential functions $P_{f}(T) \sim \exp \left(-T / T_{c}\right)$ and $P_{n}(N) \sim \exp \left(-N / N_{c}\right)$, respectively, where $T_{c} \approx 7.4$ and 10.8 for the eBay and the Korean auction data, respectively, and $N_{c} \approx 2.5$ and 5.6 for the eBay and the Korean auction data, respectively (Fig. 2).

We introduce the master equation for the bidding process as follows:

$$
n_{k}(t+1)-n_{k}(t)=w_{k-1}(t) n_{k-1}(t)-w_{k}(t) n_{k}(t)+\delta_{k, 1} u_{t},
$$

where $w_{k}(t)$ is the transition probability that a bidder, who has bid $k-1$ times up to time $t-2$, bids at time $t$ successfully. In this case, the total successful bid frequency of that agent up to time $t$ becomes $k$. Note that a bidder is not allowed to bid successively. In the master equation, we presume that the bidding pattern is similar over different items when $N(T)$ is sufficiently large. Then, $w_{k}(t)$ may be written as $w_{k}(t) \approx\langle d k / d t\rangle$ on average over different items. Empirically, we find that

$$
w_{k}(t) \approx\langle d k / d t\rangle \approx b k / t,
$$

where $b$ is estimated to be $b \approx 0.7$ for both the eBay and Korean auctions (Fig. 3). The fact that $w_{k} \propto k$ is reminiscent of the preferential attachment rule in the growing model of

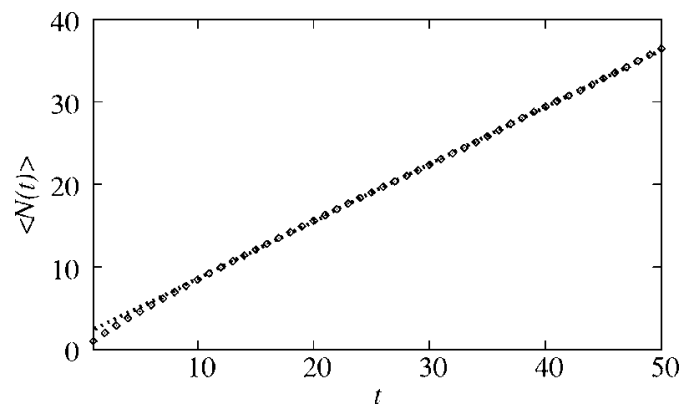

FIG. 4. Plot of $\langle N(t)\rangle$ vs $t$, averaged over different items for the eBay data. The straight line has a slope of 0.7 obtained from the least square fit. 

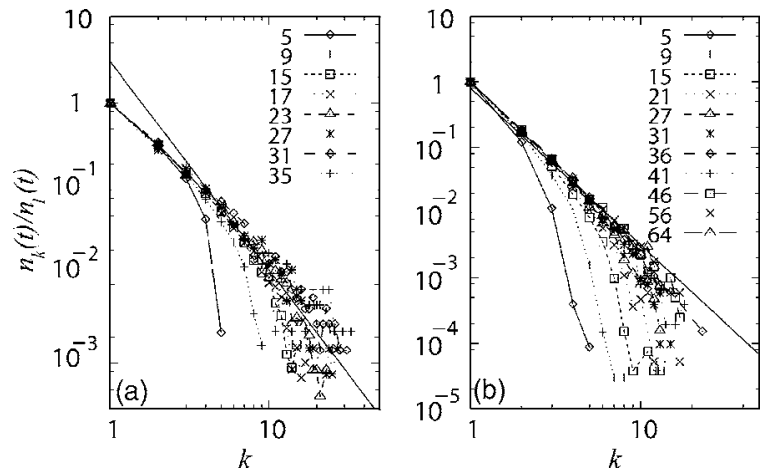

FIG. 5. Plot of $n_{k}(t) / n_{1}(t)$ vs $k$ for the eBay auction (a) and for the Korean auction (b) for various terminal times $T$. Different terminal times are represented by different symbols shown in each panel. The solid lines have a slope of -2.4 drawn for guidance.

the complex network [12]. $u_{t}$ is the probability that a new bidder makes a bid at time $t$. Using the property that $\Sigma_{k} n_{k}(t)=N(t)$, we obtain

$$
u_{t}=N(t+1)-N(t) .
$$

Next we then change the discrete equation, Eq. (3), to a continuous equation as follows:

$$
\frac{\partial n_{k}(t)}{\partial t}=-\frac{\partial}{\partial k}\left[w_{k}(t) n_{k}(t)\right]+\delta_{k, 1} u_{t},
$$

which can be rewritten as

$$
\frac{\partial n_{k}(t)}{\partial t}=-\frac{b}{t} \frac{\partial}{\partial k}\left[k n_{k}(t)\right]+\delta_{k, 1} u_{t} .
$$

When $k>1$, we use the method of separation of variables, $n_{k}(t)=I(k) T(t)$, thus obtaining

$$
\frac{\partial}{\partial k}[k I(k)]+\ell I(k)=0,
$$

where $\ell$ is a constant of separation, and

$$
\frac{\partial T(t)}{\partial t}=\frac{b \ell}{t} T(t) .
$$

Thus, we obtain

$$
n_{k}(t) \sim t^{b \ell} k^{-(1+\ell)} .
$$

When $k=1$,

$$
\frac{\partial n_{1}(t)}{\partial t}=-\frac{b}{t} n_{1}(t)+u_{t} .
$$

Next from the fact that $N=\Sigma_{k} n_{k}$, we obtain

$$
\begin{aligned}
\frac{\partial N}{\partial t} & =\sum_{k>1} \frac{\partial n_{k}}{\partial t}+\frac{\partial n_{1}}{\partial t} \\
& =\sum_{k>1}-\frac{b}{t} \frac{\partial}{\partial k}\left(k n_{k}\right)-\frac{b}{t} n_{1}+\frac{\partial N}{\partial t}
\end{aligned}
$$

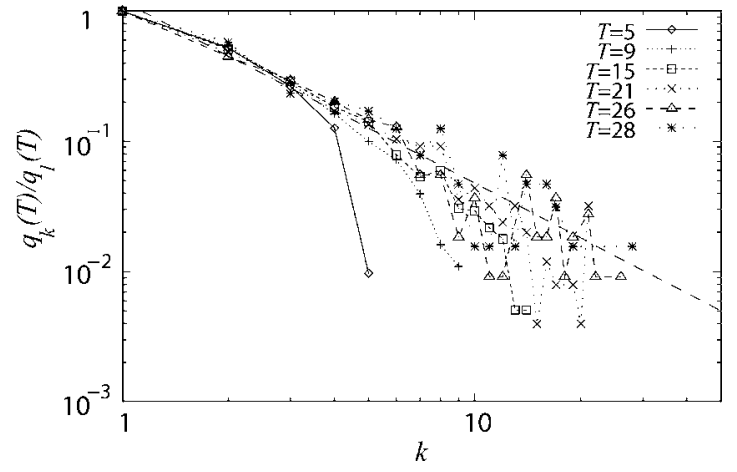

FIG. 6. Plot of the relative winning probability $q_{k}(T) / q_{1}(T)$ of the $k$-frequent bidder to that of the one-frequent bidder at the last moment vs frequency $k$. The dotted line has a slope of -1.4 drawn for guidance.

$$
=\frac{b \ell}{t}\left(N-n_{1}\right)-\frac{b}{t} n_{1}+\frac{\partial N}{\partial t} .
$$

Therefore we obtain $N(t)=(1+1 / \ell) n_{1}(t)$ and $n_{1}(t) \sim t^{b \ell}$ by using Eq. (11). Note that $N(t)<t$, and the linear relationship holds asymptotically. The linear relationship breaks down for small $t$. From the empirical data, Fig. 4, we find that $\ell b \approx 1$. Since $b \approx 0.7$ in Fig. 3 , we obtain $\ell \approx 1 / b \approx 1.4$. Therefore

$$
n_{k}(t) \sim t k^{-2.4}
$$

for large $t$, which fits reasonably with the numerical data shown in Fig. 5.

In the eBay and the Korean auctions, the winner is the last bidder in the bidding sequence. Now, we trace the bidding activity of the winner in the bidding sequence in order to find the winning strategy. To proceed, let us define $q_{k}(t+1)$ as the probability that a bidder, who has bid $k-1$ times up to time $t-1$, bids at time $t+1$ successfully. Note that a bidder is not allowed to bid successively. In this case, $q_{k}(T)$ is nothing but the probability that a $k$-frequent bidder becomes the final winner. The probability $q_{k}(t+1)$ satisfies the relation

$$
q_{k}(t+1)=\left(1-u_{t+1}\right) \sum_{j=1}^{N(t)} q_{j}(t) \frac{(k-1)\left[n_{k-1}(t)-\delta_{j, k-1}\right]}{t-j}+\delta_{k, 1} u_{t+1}
$$

with the boundary conditions $q_{1}(1)=1$ and $q_{1}(2)=1$. The first term on the right hand side of Eq. (13) is composed of three factors: (i) $1-u_{t+1}$ is the probability that one of the existing bidders bids successfully at time $t+1$, (ii) $q_{j}(t)$ means that bidding at time $t$ is carried out by the $j$-frequent bidder, and (iii) the last factor is derived from the bidding rate, Eq. (4), where the contribution by the bidder at time $t$ is excluded because he/she is not allowed to bid at time $t+1$. The second term represents the addition of a new bidder at time $t$.

The rate equation, Eq. (13), can be solved recursively. To proceed, we simplify Eq. (13) by assuming that $n_{k-1}(t)$ is significantly larger than $\delta_{j, k-1}$, which is relevant when the number of bidders is large. Then, 


$$
\begin{aligned}
q_{k}(t+1) \approx & \left(1-u_{t+1}\right) \sum_{i=1}^{N(t)} q_{i}(t) \frac{(k-1) n_{k-1}(t)}{t-i}+\delta_{k, 1} u_{t+1} \\
= & (k-1) n_{k-1}(t) \prod_{\tau=2}^{t}\left(1-u_{\tau+1}\right) \\
& \times\left(\sum_{i=1}^{\tau-1} \frac{(i-1) n_{i-1}(\tau)}{(\tau-i)}\right) q_{1}(2)+\left(1-u_{t+1}\right)(k \\
& -1) n_{k-1}(t) \sum_{\tau=3}^{t} \frac{u_{\tau}}{\tau-1} \prod_{\tau^{\prime}=\tau+1}^{t}\left(1-u_{\tau^{\prime}}\right) \\
& \times\left(\sum_{i=1}^{\tau^{\prime}-1} \frac{(i-1) n_{i-1}\left(\tau^{\prime}\right)}{\left(\tau^{\prime}-i\right)}\right)+u_{t+1} \delta_{k, 1} .
\end{aligned}
$$

Since $1-u_{t} \approx 0.3<1, q_{k}(t)$ is obtained to be

$$
q_{k}(t) \approx\left(1-u_{t-1}\right) \frac{(k-1) n_{k-1}(t-1)}{t-2}+\delta_{k, 1} u_{t}
$$

within the leading order. Considering that $n_{k}(t) \sim t k^{-2.4}$ in Eq. (12) and $u_{t}$ is constant, we obtain $q_{k}(t) \sim(t-1) k^{-1.4} /(t-2)$ for large $k$ and $t$, with a weak dependence on $t$. Thus the winning probability by the $k$-frequent bidder is simply given as

$$
q_{k}(T) \sim k^{-1.4}
$$

in the limit $t \rightarrow \infty$. This result is confirmed by the empirical data in Fig. 6.

Our analysis explicitly shows that the winning strategy is to bid at the last moment as the first attempt rather than incremental bidding from the start. This result is consistent with the empirical finding by Roth and Ockenfels [17] in eBay. According to them, the bidders who have won the most items tend to wait till the last minute to submit bids, albeit there is some probability of bids not being successfully transmitted. As evidence, they studied 240 eBay auctions and found that 89 bids were submitted in the last minute and 29 in the last ten seconds. Our result supports these empirical results.

In conclusion, we have analyzed the statistical properties of emerging patterns created by a large number of agents based on the empirical data collected from eBay.com and auction.co.kr. It is likely that the number of bidders and the winning probability decay roughly in the form of $n_{k}(t) \sim t k^{-2.4}$ and $q_{k}(t) \sim k^{-1.4}$, respectively, with bid frequency $k$.

\section{ACKNOWLEDGMENTS}

This work was supported by KRF Grant No. R14-2002-05901000-0 in the ABRL program funded by the Korean government MOEHRD and CNS in SNU (B.K.).
[1] E. van Heck and P. Vervest, Commun. ACM 41, 99 (1998).

[2] R. N. Mantegna and H. E. Stanley, An Introduction to Econophysics: Correlations and Complexity in Finance (Cambridge University Press, Cambridge, England, 2000).

[3] J. P. Bouchard and M. Potters, Theory of Financial Risks: From Statistical Physics to Risk Management (Cambridge University Press, Cambridge, England, 2000).

[4] M. H. R. Stanley, L. A. N. Amaral, S. V. Buldyrev, S. Havlin, H. Leschhorn, P. Maass, M. A. Salinger, and H. E. Stanley, Nature (London) 379, 804 (1996).

[5] D. Challet and Y.-C. Zhang, Physica A 246, 407 (1997).

[6] D. M. Pennock, S. Lawrence, C. L. Giles, and F. A. Nielsen, Science 291, 987 (2001).

[7] R. D'Hulst and G. J. Rodgers, Physica A 294, 447 (2001).

[8] I. Yang, H. Jeong, B. Kahng, and A.-L. Barabási, Phys. Rev. E 68, 016102 (2003).
[9] H. A. Simon, Biometrika 42, 425 (1955).

[10] M. Marsili and Y.-C. Zhang, Phys. Rev. Lett. 80, 2741 (1998).

[11] R. Albert and A.-L. Barabasi, Rev. Mod. Phys. 74, 47 (2002).

[12] A.-L. Barabasi and R. Albert, Science 286, 509 (1999).

[13] G. K. Zipf, Human Behavior and the Principle of Least-Effort (Addison-Wesley, Cambridge, MA, 1949).

[14] V. Pareto, Cours d'Economie Politique (Rouge, Lausanne et Paris, 1897).

[15] Y. Shoham and M. Tennenholtz, Games Econ. Behav. 35, 197 (2001).

[16] R. J. Kauffman and C. A. Wood, Proceedings of the 2000 Americas Conference on Information Systems, Long Beach, CA, August 10-13 (2000).

[17] A. E. Roth and A. Ockenfels, Am. Econ. Rev. 92, 1093 (2002). 